\title{
CONTROLE DE SEGUIMENTO DE TRAJETÓRIA DE UM ATUADOR PNEUMÁTICO ESPECIAL
}

\author{
ROZIMERLI R. M. RICHTER ${ }^{1}$, CAMILA V. ZAMBERLAN ${ }^{1}$, ANTONIO C. VALDIERO ${ }^{2}$, LUIZ A. RASIA ${ }^{2}$
}

1. Departamento de Ciências Exatas e Engenharias (DCEEng), Universidade Regional do Noroeste do Estado do Rio Grande do Sul (UNIJUÍ).

Rua São Francisco, 501 - CEP 98700-000 - Ijuí/RS, Brasil-Tel (55) 33320200

E-mails: rozymerli@hotmail.com, camilavzamberlan@yahoo.com.br

2. Departamento de Ciências Exatas e Engenharias (DCEEng), Universidade Regional do Noroeste do Estado do Rio Grande do Sul (UNIJUÍ).

Av. Rudi Franke, 540 - CEP 98280-000 - Panambi/RS, Brasil - Tel (55) 33754466

E-mails: valdiero@unijui.edu.br, rasia@unijui.edu.br

\begin{abstract}
This work presents the mathematical model of a special pneumatic cylinder considering the main nonlinear characteristics system and trajectory position control. The pneumatic actuator prototype and design were developed at the in NIMeP/UNIJUÍ with financial support by CELPE in ANEEL P\&D Program. Computer simulations were performed using the software MatLab / Simulink to compare these results with those achieved in the experimental test bench.
\end{abstract}

Key - words: pneumatic actuator for robotics, proportional control, trajectory planning.

Resumo: Este trabalho apresenta o modelo matemático de um cilindro pneumático especial considerando-se as principais características não lineares do sistema e o controle de seguimento de trajetória de posição. O projeto e o protótipo do atuador pneumático utilizado foram desenvolvidos no NIMeP/UNIJUÍ com suporte financeiro da CELPE no âmbito do Programa P\&D da ANEEL. As simulações computacionais foram realizadas com auxílio do software MatLab/Simulink a fim de comparar estes resultados com os testes realizados na bancada experimental.

Palavras - chave: atuador pneumático para robótica, controle proporcional, planejamento de trajetória.

\section{Introdução}

Este trabalho apresenta o modelo matemático de um atuador pneumático especial com o curso do cilindro de 2,5 metros e considerando-se as principais características não lineares presentes no sistema, assim como o projeto de uma estratégia de controle proporcional para o seguimento de uma dada trajetória desejada.

Atuadores pneumáticos são sistemas muito atrativos para diversas aplicações, em especial na robótica, porque eles têm a vantagem de baixo custo, leveza, durabilidade e são limpos quando comparados com os atuadores hidráulicos, também possuem facilidade de manutenção, têm boa relação força/tamanho e flexibilidade de instalação e, além disso, o ar comprimido está disponível na maioria das instalações industriais (GUENTHER et al., 2006; WEICKGENANNT et al., 2010; QIONG et al., 2011). Os servoposicionadores pneumáticos também apresentam menor risco de contaminação ambiental e de operação em relação aos sistemas hidráulicos, visto que, se ocorrer no sistema hidráulico um vazamento de óleo, isso poderá gerar sérios danos ambientais, ainda maiores se este óleo pode ser inflamável, destaca Suzuki (2010).

Em despeito dessas vantagens, sistemas de posicionamento pneumático possuem algumas características indesejáveis as quais limitam o uso destes em aplicações que requerem uma resposta precisa (GUENTHER et al., 2006; ALLGAYER,
2011).Estas características indesejáveis derivam da alta compressibilidade do ar (WEICKGENANNT et al., 2010) e das não-linearidades presentes em sistemas pneumáticos, tais como o comportamento não linear da vazão mássica nos orifícios da válvula e sua zona morta (VALDIERO et al., 2011), além do atrito nas vedações do cilindro linear (ANDRIGHETTO et al., 2006).

$\mathrm{Na}$ seção 2 apresenta-se o propósito do trabalho. A metodologia é expressa na seção 3 . Uma breve descrição do atuador pneumático é apresentada na seção 4 . Na seção 5 ocorre a modelagem matemática do sistema considerando as características não lineares. A seção 6 mostra os resultados obtidos na modelagem matemática e nas simulações computacionais, validados nos testes experimentais para o controle de seguimento de trajetória. $\mathrm{Na}$ sequência apresentam-se as conclusões na seção 7 .

\section{Propósito}

Esta trabalho é um dos resultados do projeto de pesquisa e desenvolvimento desenvolvido no âmbito do Programa de Pesquisa e Desenvolvimento Tecnológico do Setor de Energia Elétrica regulado pela ANEEL (código ANEEL PD-0043-0311/2011) e trada da pesquisa em pneumática para aplicação em um equipamento para poda de árvores. $\mathrm{O}$ estudo e modelagem 
matemática de um atuador pneumático de longo curso de movimentação permite o afastamento adequado do operário ou da base fixa do manipulador robótico em relação ao galho que será cortado, evitando assim os graves acidentes, especialmente nas redes de alta tensão, desta forma o comportamento dinâmico destes equipamentos precisam ser modelado.

O problema da poda é que a mesma requer dos equipamentos utilizados confiabilidade nos movimentos desejados. Entretanto, há vários fatores que dificultam a obtenção de bom desempenho com repetições, a posição da haste do cilindro pneumático é uma delas (RICHTER, 2013).

Assim, este trabalho apresenta alguns dos resultados do controle de posição com seguimento de trajetória de um atuador pneumático para uma dada aplicação em um equipamento florestal para uma faixa de trabalho em torno de 2,5 metros de curso.

\section{Metodologia}

Para análise dos resultados utilizou-se simulações computacionais realizadas no software MatLab/Simulink com método de integração Runge Kutta, passo fixo de $0.0001 \mathrm{~s}$, com dados dos testes experimentais realizados em uma bancada do
Núcleo de Inovação e Mecanização da Poda (NIMeP), pertencente ao Departamento de Ciências Exatas e Engenharias (DCEEng) no Campus Panambi da Universidade Regional do Noroeste do Estado do Rio Grande do Sul (UNIJUÍ).

$\mathrm{Na}$ bancada experimental, onde se realizou os testes, possui um sistema de controle e de aquisição de dados composto por uma placa dSPACE 1104 responsável pela captura e armazenagem dos dados da bancada de testes, a qual utiliza a integração dos softwares MatLab/Simulink e ControlDesk permitindo a captura, controle e manipulação dos dados em tempo real através da construção de uma interface gráfica, o que possibilita a análise detalhada dos resultados obtidos.

\section{Descrição do Atuador Pneumático}

O servoposicionador pneumático é um sistema dinâmico composto por uma servoválvula de controle direcional, um cilindro pneumático linear especial de dupla ação e haste simples, e o sistema de controle. Este servo sistema permite posicionar uma carga em um determinado ponto do curso do atuador ou seguir uma trajetória variável em função do tempo. A Figura 1 ilustra através de um desenho esquemático o servoposicionador pneumático.

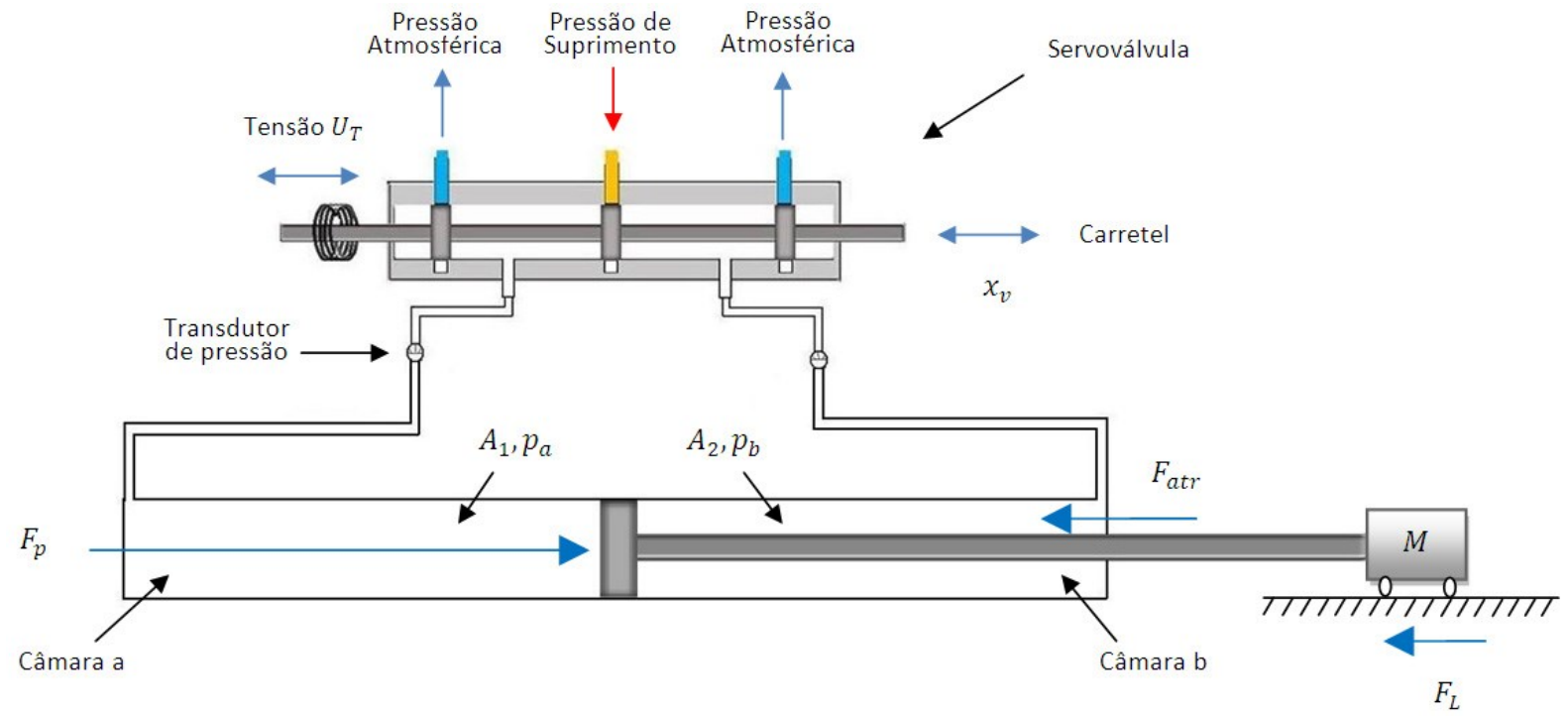

Figura 1- Desenho esquemático de um servoposicionador pneumático linear. Fonte: Própria autora

O servoposicionador pneumático funciona com o ar comprimido que é fornecido à servoválvula a uma dada pressão de suprimento $\left(p_{s}\right)$ previamente regulada. Durante a operação, o sinal de controle $U_{T}$ energiza o solenóide da válvula de modo que uma força magnética resultante é aplicada no carretel da válvula, produzindo o deslocamento do carretel. $\mathrm{O}$ deslocamento do mesmo abre os orifícios de controle para que uma câmara do cilindro linear seja ligada a linha de pressão de suprimento e a outra seja ligada à pressão atmosférica $\left(p_{a t m}\right)$. Desta forma, produzindo uma diferença de pressão nas câmaras do cilindro linear, dando origem a uma força resultante movendo a massa $M$ preza a haste deste cilindro, em um deslocamento no sentido positivo 
ou negativo $y$, dependendo do sinal de entrada. Esta força gerada pelo atuador pneumático é dada pelo produto da área do êmbolo do cilindro pela diferença de pressão nas câmaras e é chamada de força pneumática.

\section{Modelo matemático do Atuador acionado Pneumaticamente}

O modelo não linear de $5^{\mathrm{a}}$ ordem a seguir é descrito na forma de variáveis de estado, considerando $y_{1}=y, y_{2}=\dot{y}, y_{3}=p_{a}, y_{4}=p_{b}$ e $y_{5}=z$ :

$$
\begin{gathered}
\dot{y}_{1}=y_{2} \\
\dot{y}_{2}=-\frac{F_{a t r}\left(y_{5}, y_{2}\right)}{M}+\frac{A_{1}}{M} y_{3}-\frac{A_{2}}{M} y_{4} \\
\dot{y}_{3}=-\frac{\gamma A_{1}}{V_{a 0}+A_{1} y_{1}} y_{2} y_{3}+\frac{\gamma R T}{V_{a 0}+A_{1} y_{1}} q_{m a}\left(U_{T}, y_{3}\right) \\
\dot{y}_{4}=\frac{\gamma A_{2}}{V_{b 0}-A_{2} y_{1}} y_{2} y_{4}-\frac{\gamma R T}{V_{b 0}-A_{2} y_{1}} q_{m b}\left(U_{T}, y_{4}\right) \\
\dot{y}_{5}=y_{2}-\alpha\left(y_{5}, y_{2}\right) \frac{\sigma_{0}}{g_{s s}\left(y_{2}\right)} \operatorname{sgn}\left(y_{2}\right) y_{5}
\end{gathered}
$$

onde $y_{1}$ é a posição do êmbolo, $y_{2}$ é a velocidade, $y_{3}$ e $y_{4}$ as pressões nas câmaras $a$ e $b$ do cilindro, e $y_{5}$ é a dinâmica das microdeformações, $M$ é a massa acoplada, $A_{1}$ e $A_{2}$ são as áreas do êmbolo das câmaras $a$ e $b$ do cilindro, $T$ a temperatura do ar de suprimento, $R$ é a constante universal dos gases, $\gamma=C_{p} / C_{v}$ é a relação entre os calores específicos do ar, onde $C_{p}$ e $C_{v}$ são os calores específicos do ar sob pressão constante e a volume constante, respectivamente, $V_{a 0}$ e $V_{b 0}$ são os volumes mortos nas câmaras $a$ e $b, q_{m a}$ e $q_{m b}$ são as vazões mássicas nas câmaras $a$ e $b$ do cilindro, $F_{a t r}$ é a força de atrito, $\sigma_{0}$ representa o coeficiente de rigidez das deformações microscópicas entre as superfícies de contato, $g_{s s}(\dot{y})$ representa uma função positiva que descreve parte das características do atrito em regime permanente e a função $\alpha(z, \dot{y})$ de acordo com (DUPONT et al., 2000; RITTER, 2010) foi incorporada ao modelo LuGre e é utilizada para obter a representação da stiction, ou seja, o regime estático em velocidades baixíssimas. A modelagem matemática detalhadamente encontra-se em Richter (2013).

\section{Resultados}

Nesta seção são apresentados os resultados da modelagem matemática da dinâmica de atuadores

\begin{tabular}{|c|c|c|}
\hline $\begin{array}{c}\text { Descrição do } \\
\text { Parâmetro }\end{array}$ & Simbologia & Valor \\
\hline Pressão de suprimento & $p_{s}$ & $7 \times 10^{5} \mathrm{~Pa}$ \\
\hline Pressão atmosférica & $p_{a t m}$ & $1 \times 10^{5} \mathrm{~Pa}$ \\
\hline $\begin{array}{c}\text { Constante universal dos } \\
\text { gases }\end{array}$ & $R$ & $287 \mathrm{Jkg} / \mathrm{K}$ \\
\hline Temperatura do ar & $T$ & $293 K$ \\
\hline $\begin{array}{c}\text { Relação entre os } \\
\text { calores específicos do } \\
\text { ar }\end{array}$ & $\gamma$ & $\begin{array}{c}1.4 \\
\text { Adimensional }\end{array}$ \\
\hline Área do êmbolo & $A_{1}$ & $4.1 \times 10^{-3} \mathrm{~m}^{2}$ \\
\hline $\begin{array}{c}\text { Área do êmbolo } \\
\text { descontada a haste }\end{array}$ & $A_{2}$ & $3.0 \times 10^{-3} \mathrm{~m}^{2}$ \\
\hline $\begin{array}{l}\text { Volume morto na } \\
\text { câmara } a\end{array}$ & $V_{a 0}$ & $5.1 \times 10^{-3} \mathrm{~m}^{3}$ \\
\hline $\begin{array}{c}\text { Volume morto na } \\
\text { câmara } b\end{array}$ & $V_{b 0}$ & $4.0 \times 10^{-4} \mathrm{~m}^{3}$ \\
\hline Massa acoplada & $M$ & $5 \mathrm{~kg}$ \\
\hline
\end{tabular}
pneumáticos, destacando-se o projeto de controle no seguimento de trajetória desejada. Os valores dos parâmetros do atuador pneumático estão descritos nas Tabelas 1 e 2 .

A identificação dos parâmetros estáticos e dinâmicos do atrito são provenientes de diversos experimentos em malha aberta, descritos minuciosamente em Richter et al. (2013).

Tabela 2 - Parâmetros do Atrito.

\begin{tabular}{c|c|c}
\hline Descrição do Parâmetro & Simbologia & Valor \\
\hline Atrito estático & $F_{s}$ & $100 \mathrm{~N}$ \\
\hline Atrito Coulomb & $F_{c}$ & $70 \mathrm{~N}$ \\
\hline Coeficiente de Arraste & $C_{d}$ & $4546.7 \mathrm{Ns} / \mathrm{m}^{2}$ \\
\hline Velocidade Stribeck & $\dot{y}_{s}$ & $0.01 \mathrm{~m} / \mathrm{s}$ \\
\hline $\begin{array}{c}\text { Coeficiente de rigidez das } \\
\text { microdeformações }\end{array}$ & $\sigma_{0}$ & $1.4 \times 10^{6} \mathrm{~N} / \mathrm{m}$ \\
\hline $\begin{array}{c}\text { Coeficiente de } \\
\text { amortecimento das } \\
\text { microdeformações }\end{array}$ & $\sigma_{1}$ & $50 \mathrm{Ns} / \mathrm{m}$ \\
\hline
\end{tabular}

Um dos métodos mais simples de controle de um sistema comentado por Soboczyk (2009) baseia-se apenas em uma realimentação proporcional (P) do desvio de seguimento da variável cujo valor se deseja controlar. Desta forma, o sinal de controle utilizado é simplesmente o erro de seguimento $e(t)$ multiplicado por uma constante, isto é, a saída $\left(U_{T}\right)$ do controlador é um sinal diretamente proporcional ao erro de posição $\left(y-y_{d}\right)$. Este erro é a diferença algébrica entre a posição medida e a posição desejada, ou seja:

$$
U_{T}=k_{p}\left(y-y_{d}\right)
$$


onde $k_{p}$ é o ganho proporcional.

Este controle tem por objetivo direcionar o sistema a um ponto fixo, não devendo ser brusco, a fim de não prejudicar ou danificar o sistema. Para isso o planejamento da trajetória se dá pela necessidade de obter as informações de desempenho do posicionamento da haste deste cilindro nos intervalos (trechos) de seguimento como nas paradas (regulação), isto significa que, existe um posicionamento inicial, intermediário e final, sempre levando em conta as paradas. Desta forma escolheu-se a trajetória polinomial de $7^{\mathrm{a}}$ ordem (equação 7), a qual atende a característica de suavidade, além de permitir a especificação das condições iniciais e finais para a posição desejada.

$$
\begin{gathered}
y_{d p}(t)=a_{7} t^{7}+a_{6} t^{6}+a_{5} t^{5}+a_{4} t^{4}+a_{3} t^{3}+a_{2} t^{2} \\
+a_{1} t+a_{0}
\end{gathered}
$$

A equação (8) mostra a trajetória de posição desejada a qual possui todos os intervalos com duração de $I$ segundos com valor de 15 segundos. Tendo um intervalo de parada inicial, chamado de regulação, seguido de um intervalo de subida até a posição intermediária do cilindro, caracterizado por um polinômio de $7^{\mathrm{a}}$ ordem, $y_{d p}(t)$, na posição intermediária novamente ocorre um intervalo de regulação. $\mathrm{Na}$ sequência um intervalo de subida até a posição superior, posteriormente um intervalo de regulação, e assim com as mesmas características ocorre no recuo, respectivamente um intervalo de descida até a posição intermediária do cilindro, um intervalo de regulação, uma nova descida até a posição inferior e para finalizar o período da trajetória um intervalo de regulação.

$$
y_{d}(t)=\left\{\begin{array}{cc}
y_{\min } & t<I \\
y_{d p}(t-I)-\left|y_{\min }\right| & I \leq t \leq 2 I \\
0 & 2 I<t<3 I \\
y_{d p}(t-3 I) & 3 I \leq t \leq 4 I \\
y_{\max } & 4 I<t<5 I \\
-y_{d p}(t-5 I)+y_{\max } & 5 I \leq t \leq 6 I \\
0 & 6 I<t<7 I \\
-y_{d p}(t-7 I) & 7 I \leq t \leq 8 I \\
y_{\min } & 8 I<t<9 I
\end{array}\right.
$$

onde $y_{\min }$ e $y_{\max }$ apresentam o mesmo valor devido o cilindro estar centrado, ou seja, $y_{\min }=$ $y_{\text {max }}=y_{f}=1,2415 \mathrm{~m}$. O polinômio resultante descreve-se na equação (9) e o detalhamento da determinação dos coeficientes do polinômio em Richter (2013).

$$
\begin{aligned}
y_{d p}(t)=\left(-0,0145 t^{7}\right. & +0,762 t^{6}-13,733 t^{5} \\
& \left.+85,832 t^{4}\right) 10^{-5}
\end{aligned}
$$

Como resultado do planejamento de trajetória com controle proporcional, a escolha do ganho proporcional necessita ser conveniente, a fim de que o sistema não se torne instável, causando sérios prejuízos e a redução da vida útil do atuador pneumático. Como desgaste da servoválvula, das vedações do cilindro, aparecimento de vazamentos, possível danificação no sensor de posição, o qual está acoplado dentro da haste do cilindro, além de causar riscos ao ser humano que está realizando o manuseio.

Levando em consideração o limite do ganho do controlador $k_{p}=70$, preservando a estabilidade do sistema, as Figuras 2 e 3 mostram os resultados do planejamento da trajetória polinomial com controle proporcional.

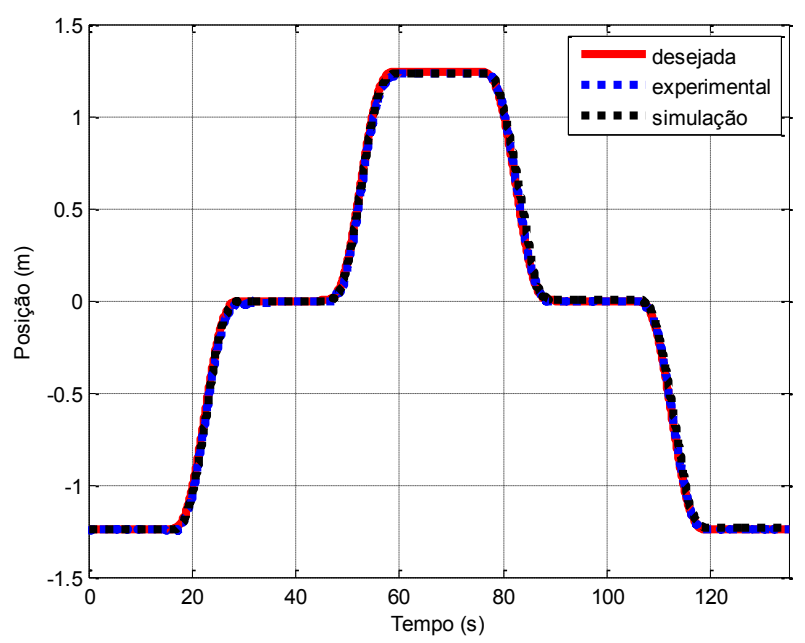

Figura 2 - Posição do sistema para trajetória polinomial com controlador proporcional.

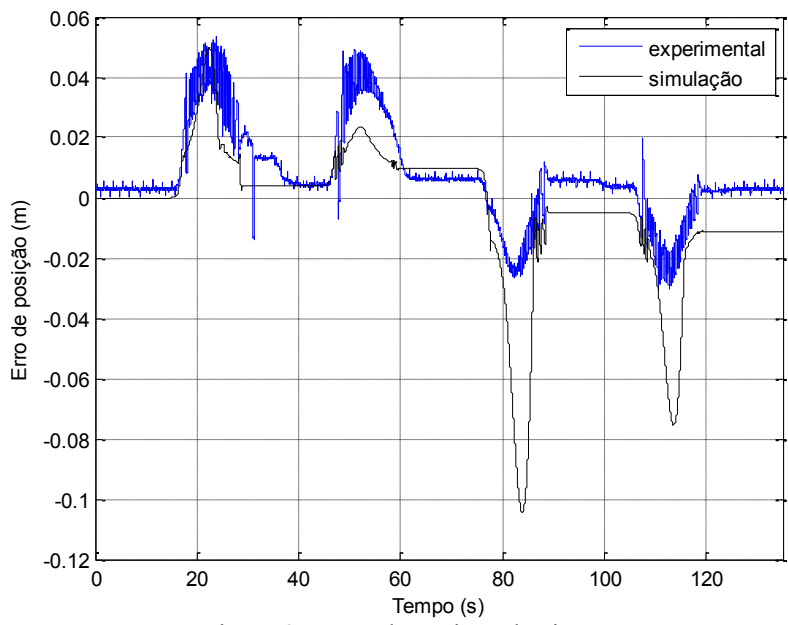

Figura 3 - Erro de posição do sistema.

\section{Conclusão}

Apresentou-se o modelo matemático para o atuador pneumático, o qual descreve o comportamento dinâmico de uma bancada de simulação do posicionamento para uma dada aplicação em um equipamento florestal acionado pneumaticamente, incluindo as principais características não lineares do mesmo. A 
comparação dos resultados computacionais e experimental ilustra a validação do modelo matemático proposto para estudo da dinâmica de atuadores pneumáticos. O controle proposto sendo clássico mostrou-se eficiente para os objetivos estabelecidos. Os erros observados podem ser reduzidos consideravelmente na utilização de controladores que tenham mais recursos. Estes resultados obtidos serão utilizados no aperfeiçoamento do projeto P\&D de título "Desenvolvimento de Solução Mecanizada para a Poda de Árvores com Incidência sobre Componentes Energizados de Linhas e Redes Aéreas de Distribuição de Energia Elétrica”.

\section{Agradecimentos}

Os autores agradecem a CAPES pelo apoio na forma de bolsas de mestrado; a UNIJUÍ pelo apoio e incentivo na realização da pesquisa, e também pelo apoio financeiro da CELPE (Companhia Energética de Pernambuco) no âmbito Programa P\&D da ANEEL (código ANEEL PD-00430311/2011) que permitiu a implantação da estrutura laboratorial do NIMeP (Núcleo de Inovação e Mecanização da poda).

\section{Referências Bibliográficas}

ALLGAYER, Renan Schimidt. Desenvolvimento de um manipulador robótico cilíndrico acionado pneumaticamente. 2011. 99f. Dissertação (Mestrado em Engenharia Mecânica) - Universidade Federal do Rio Grande do Sul, Porto Alegre, 2011.

ANDRIGHETTO, P. L. ; VALDIERO, A. C. ; CARLOTTO, L. Study of the friction behavior in industrial pneumatic actuators In: ABCM Symposium Series in Mechatronicsed.Rio de Janeiro : ABCM Associação Brasileira de Engenharia e Ciências Mecânicas, 2006, v.2, p. 369-376.

DUPONT, P.; ARMSTRONG, B.; HAYWARD, V. Elasto-plastic friction model: Contact compliance and stiction. In: ACC, American Control Conference, Chicago, Illinois, Mar. 2000, p.1072-1077.

GUENTHER, Raul; PERONDI, Eduardo A.; DEPIERI, Edson R. and VALDIERO, Antônio C. Cascade controlled pneumatic positioning system with LuGre model based friction compensation. J. Braz. Soc. Mech. Sci. \& Eng. [online]. 2006, vol.28, n.1, p. 4857. ISSN 1678-5878.

QIONG, W.; JIAO, Z. Modeling and analysis of pneumatic loading system. International
Conference In: Fluid Power and Mechatronics (FPM), 2011, p. $642-646$.

RICHTER, R. R. M. Modelagem matemática e controle de posição de um atuador acionado pneumaticamente. 2013. 109f. Dissertação (Mestrado em Modelagem Matemática) - Universidade Regional do Noroeste do Estado do Rio Grande do Sul, Ijuí, 2013.

RICHTER, R. R. M; ZAMBERLAN, C. V.; VALDIERO, A. C.; RASIA, L. A. Friction dynamics mathematical modeling in special pneumatic cylinder. 22nd International Congress of Mechanical Engineering (COBEM 2013). November 3-7, 2013, Ribeirão Preto, SP, Brazil. (Submetido)

RITTER, C. S. Modelagem matemática das características não lineares de atuadores pneumáticos. 2010. 87f. Dissertação (Mestrado em Modelagem Matemática) Universidade Regional do Noroeste do Estado do Rio Grande do Sul, Ijuí, 2010.

SOBCZYK, Mário Roland. Controle em Cascata e a Estrutura Variável com Adaptação de Parâmetros e Compensação de Atrito de um Servoposicionador Pneumático. 2009, 222f Tese (Doutorado em Engenharia Mecânica) - Programa de Pós-graduação em Engenharia Mecânica, Universidade Federal do Rio Grande do Sul, Porto Alegre, 2009.

SUZUKI, Ricardo Murad. Controle baseado em linearização por realimentação dos estados aplicado a um servoposicionador pneumático. 2010. 110f. Dissertação (Mestrado em Engenharia Mecânica) Universidade Federal do Rio Grande do Sul, Porto Alegre, 2010.

VALDIERO, A. C. ; RITTER, C.S. ; RIOS, C.F. ; RAFIKOV, Marat .NonLinear Mathematical Modeling in Pneumatic Servo Position Applications. Mathematical Problems in Engineering (Online), v. 2011, p. 1-16, 2011. DOI: 10.1155/2011/472903

WEICKGENANNT, Martin; ZIMMERT, Nico; KLUMPP, Simon; SAWODNY, Oliver. Application of SDRE control to servopneumatic drives. IEEE International Conference on Control Applications (CCA), 2010, p. $1725-1730$. 